\title{
Diethyl Azelate for the Treatment of Brown Recluse Spider Bite, a Neglected Orphan Indication
}

\author{
ROBERT T. STREEPER* and ELZBIETA IZBICKA* \\ New Frontier Labs LLC, San Antonio, TX, U.S.A.
}

\begin{abstract}
Background/Aim: Brown recluse spider bite releases hemolytic and cytotoxic phospholipase $D$ to the wound that may cause necrosis or even death. We examined diethyl azelate (DEA), a plasma membrane fluidizer with a broad range of immunomodulatory activities, as a potential treatment for the brown recluse spider bite. Materials and Methods: Topical DEA was used in emergency to treat brown recluse spider bites in a human subject. We subsequently evaluated the effects of DEA on hemolysis induced by the brown recluse spider venom, recluse recombinant phospholipase D (rPLD), and venoms from honey bee and moccasin snake, and on phospholipase A2 activity in the bee and snake venoms and in human urine. Results: Topical DEA resolved the consequences of human brown recluse spider envenomation in two weeks. In vitro, DEA inhibited hemolysis caused by the brown recluse spider venom and rPLD and suppressed phospholipase A2 activity in a dosedependent manner. Conclusion: DEA is a promising novel therapy for the brown recluse spider bite and perhaps even unrelated envenomations involving PLDs.
\end{abstract}

Our atavistic fear of spiders is well justified. Venomous spiders are found all over the world, not only in tropics but also in temperate zones. The most common venomous spider in the United States is brown recluse spider Lososceles reclusa and its habitat has been expanding from the southcentral regions further north (1). L. reclusa bite can cause necrotic arachnidism (loxoscelism) (2). Despite the existence of a diagnostic test and a mnemonic NOT RECLUSA device (3), the actual loxoscelism cases may be overestimated

This article is freely accessible online.

*These Authors contributed equally to this work.

Correspondence to: Elzbieta Izbicka, New Frontier Labs LLC, 900 NE Loop 410, suite D-119, San Antonio, Texas, U.S.A. Tel: +1 2107256868,e-mail: eizbicka.g4@gmail.com

Key Words: Azelate, fatty acid ester, brown recluse, spider bite. because other conditions such as bacterial, viral or fungal infections, insect bites, and medical conditions that affect blood circulation can mimic the effects of the bite of a brown recluse spider (3). Confirmed cases of loxoscelism are relatively rare in the United States (4). With approximately 3,500 annual cases of moderate to severe envenomations (5), loxoscelism is considered an orphan disease. However, since severe systemic loxoscelism is associated with $3.5 \%$ mortality and may cause rapid fatality (6), there is an urgent need for a specific treatment despite scarcity of cases.

L. reclusa venom is cytotoxic and hemolytic. Among multiple toxins in the venom, phospholipase D (PLD) is largely responsible for tissue destruction and hemolysis (7). In model systems, PLD alone can reproduce most of the symptoms of loxoscelism (8). PLD is a potent endothelial cell agonist and promotes secretion of pro-inflammatory cytokines, chemokines and metalloproteinases (9). PLD toxin targets fibroblasts, platelets and erythrocytes, and histamine may potentiate its toxicity (8).

PLD toxins are widespread in the animal kingdom. PLDs in the SicTox spider gene family are produced by Loxosceles, Sicarius and Hexophtalma spiders, and are also present in tick saliva, scorpion venom and exotoxins of pathogenic bacteria and fungi (10). PLD toxins belong to the protein domain family of GDPD-like sphingomyelinase D/PLDs (11). Among the PLD toxins these of L. reclusa are the best characterized. PLD produces soluble choline and lipid cyclic phosphates by transphosphatidylation of lysophosphatidic acid. These potentially pathogenic lipid cyclic phosphates may cause disruption of lipid rafts (12) and act as neo-antigens (7).

To date there are no approved drugs for loxoscelism. Largely symptomatic standard of care may include nonsteroidal anti-inflammatory drugs, corticosteroids, and antibiotics. A horse polyvalent antiserum available in Brazil and other South America countries must be administered within $24 \mathrm{~h}$ of envenomation. The efficacy of other nonspecific treatments such as dapsone is questionable. Current efforts in drug development for loxoscelism include complement inhibitors, natural oils, small molecule inhibitors of PLD, tetracycline and others (13). 
Venoms of various spiders, scorpions, bees and snakes contain secretory phospholipases A2 (sPLA2). The sPLA2 enzymes are also found in species as diverse as bacteria and humans (14). PLA2s break down phospholipids to fatty acids including arachidonic acid and induce pain and inflammation in mammals. Upregulation of sPLA2 may promote vascular inflammation in coronary artery disease and acute coronary syndrome. Both PLDs and PLA2s belong to the large family of phospholipases, ubiquitous enzymes that catalyze the hydrolysis of phospholipids into fatty acids. The phospholipases, typically heterodimeric proteins, differ from other enzymes that bind water-soluble substrates (14), as phospholipase substrates reside in cell plasma membranes.

We have identified a novel class of drugs that we refer to as Membrane Active Immunomodulators (MAIMs) that use the entire cell plasma membrane as their target $(15,16)$. MAIMs alter membrane fluidity and shift the innate feedback mechanism regulating fluidity homeostasis mechanism, which we have named adaptive membrane fluidity modulation (AMFM). In studies on MAIMs, such as diethyl azelate (DEA) and related esters, we have shown that the azelates modified plasma membrane fluidity in a structure-related manner and affected the activities of membrane proteins relevant for innate immune responses, resulting in altered patterns of signaling molecules produced by human and animal cells. In particular, DEA displayed a broad range of activities in seemingly unrelated cases. The ester modulated activities of pathogen-associated molecular pattern receptors and mitigated effects of bacterial toxins such as cholera and anthrax lethal toxin. Furthermore, DEA demonstrated efficacy against antibiotic resistant Staphylococcus aureus (15) and Mycobacterium ulcerans (manuscript in preparation). In a human study in overweight males with insulin resistance, orally administered DEA significantly reduced fasting glucose and insulin in these subjects, and improved the diagnostic lipid ratios (17).

Although DEA is not yet available as a drug, its parent molecule, azelaic acid, has been used for decades in dermatology for various skin conditions (18). The maximum dose of topical azelaic acid is $20 \%$ due to its acidity. However, DEA being an ester has an advantage over free acid in topical applications since it can be used at the full strength of $100 \%$.

This study described a new application for DEA as a treatment for the brown recluse spider bite based on two human case studies and supporting laboratory experiments.

\section{Materials and Methods}

Chemical synthesis and reagents. All reagents for the chemical synthesis were sourced from Sigma Chemical (St. Louis, MO, USA). Alkyl azelaic acid esters (azelates) were synthesized from azelaic acid and respective alcohols (methyl, ethyl, propyl, isobutyl, 1-pentyl, 3-pentyl, and cyclohexyl) using the standard acidcatalyzed esterification to produce dimethyl azelate (DMA), diethyl azelate (DEA), di-isopropyl azelate (DiPA), di-isobutyl azelate (DiBU), di-(1-pentyl) azelate (D1PA), di-(3-pentyl) azelate (D3PA), and dicyclohexyl azelate (DCHA) $(15,17)$. Lyophilized L. reclusa venom (LOX) was purchased from SpiderPharm (Yarnell, AZ, USA). Immediately prior to the assay, LOX was reconstituted in $20 \%$ glycerol in phosphate buffered saline. Full length recombinant L. intermedia phospholipase D LiSicTox-alphaIA1a protein expressed in E. coli (part \#MBS1258368), further referred to as PLD, was from MyBiosource Inc, (San Diego, CA, USA). Lyophilized venoms from honey bee (Apis cerana) and water moccasin snake (Agkistrodon piscivorus) were obtained from Kentucky Reptile Zoo (Slade, KY, USA), and Jinan Grace Industry (Jinan, PR China), respectively. Whole blood and urine specimens were sourced from a healthy male donor.

Isolation of erythrocytes from human peripheral blood. Fresh human peripheral blood mononuclear cells (PBMC) were obtained from a healthy volunteer ( $\mathrm{RS}$, the co-author of this manuscript). Whole peripheral blood was collected into BD Vacutainer CPT Cell Preparation Tubes (Beckton Dickinson, Franklin Lakes, NJ, USA) and centrifuged at $500 \times g$ at room temperature. Pelleted erythrocytes were washed twice each with $150 \mathrm{mM} \mathrm{NaCl}$ and with phosphate buffered saline (PBS) and then reconstituted in 50-fold excess PBS for hemolysis assay.

Hemolysis assay. The assay using human erythrocytes followed an established procedure (19). Immediately prior to the assay, LOX was reconstituted in $20 \%$ glycerol in PBS and stored on ice. rPLD supplied as frozen stock in $10 \mathrm{mM}$ Tris-HCl, $1 \mathrm{mM}$ EDTA, $20 \%$ glycerol, was thawed on ice. The lysis assay was performed in 96-wells in a total volume of $200 \mu \mathrm{l}$. LOX, rPLD, bee and moccasin snake venoms, and human urine were added as $20 \times$ concentrated stocks to the erythrocyte suspension. Triton X-100 (from $0.04 \%$ to $5 \%$ ) was used as a positive control. Incubations were performed at room temperature for $60 \mathrm{~min}$ and the absorbance at $400 \mathrm{~nm}$ was measured using Tecan GENios microwell plate reader. The assays were performed in triplicate (controls) or duplicate (treatment groups).

Topical DEA treatment of brown recluse spider bite. The investigator on the study (RS) was bitten once on the left forearm and a year later got two adjacent bites in the neck. In both cases, topical DEA was applied daily at approximately 4 to $8 \mathrm{~h}$ intervals for 14 days. Progression of the treatment was documented by photography. Images of the affected areas were normalized to the same size and converted to JPEG files. The arm bite images were processed using Image J software version $1.53 \mathrm{e}$ and converted to inverse grayscale. For the neck bite, inflamed red areas surrounding the bites, visible as elevated lesions, were outlined on the images and quantified using ImageJ software as above.

Phospholipase A2 assay. The assay kit for secretory phospholipase A2 (sPLA2) was purchased from Cayman Chemical (Ann Arbor, MI, USA) and the assay was performed according to the supplier's manual. DEA was diluted in DMSO and the venoms were diluted in PBS to obtain linear kinetics of PLPA2 activity for $10 \mathrm{~min}$. Prior to initialing the enzymatic reaction, the free thiol detecting reagent 5,5'-dithio-bis-(2-nitrobenzoic acid), the assay buffer and the test 
articles or vehicle controls were delivered to the microwell assay plate and gently mixed at room temperature on a horizontal mixer for 15 min unless specified otherwise. The reaction was initiated by addition of the substrate followed by gentle mixing. The absorbance at $400 \mathrm{~nm}$ was measured continuously in ten 1-min cycles using Tecan GENios microwell plate reader. The measurements were performed in duplicates in two separate experiments. For data analysis, the background absorbance was subtracted and the linear initial velocities over the first 5 min were plotted for data analysis.

Ethical statement. The self-experiment on human subjects and phlebotomy procedure were in accordance with protocols number NF-009-016 and NF-001-018 approved by the Institutional Review Board at New Frontier Labs LLC, San Antonio, TX, USA and with the Helsinki Declaration of 1975, as revised in 1983.

Statistical analysis. Excel v.2104 (Microsoft Office 2019) statistical functions were used; $p$-values of $<0.05$ were considered statistically significant.

\section{Results}

DEA resolved consequences of the brown recluse bite. The utility of DEA in the treatment of brown recluse spider bite came about via serendipity when the co-author of this manuscript (RS) experienced accidental bite on the right forearm. Knowing that there is no curative treatment for the recluse bite and given the documented safety and efficacy of oral DEA (17), the victim chose compassionate emergency use of topical DEA. The DEA treatment topically twice a day was initiated within $4 \mathrm{~h}$ of the incident. During the first day the initially painless wound area began to swell and became warm and achy. By the second day the arm had approximately twice the pre-bite circumference and was mildly uncomfortable. By the fourth day the swelling and discomfort had largely subsided. The injury resolved without complications in 10 days. Representative images of the arm taken over the course of 7 days are shown in Figure 1. The color scale images are presented in the left column, inverted colors in the right column, and the site of the bite is marked with an arrow. The disappearance of the inflammatory zone at the bite site that is no longer detectable at $72 \mathrm{~h}$ and later times underscores the efficacy of the treatment.

Approximately one year after the first incident the investigator suffered accidental two neck bites by brown recluse. The DEA treatment was initiated immediately after the bite. Representative images of the neck taken over the course of 14 days are shown in Figure 2. A magnified image of the bite area on Day 1 at $4 \mathrm{~h}$ post-bite is shown in Figure $2 \mathrm{~A}$, and the two bite sites are indicated by arrows. Corresponding results of image analysis over the course of 14 days are presented in Figure 2B. The inflamed red area around the two sites of the bite on the neck increased rapidly as shown on the first recorded image at $1 \mathrm{~h}$ post-bite reaching the maximum on Day 2 at 26 h post-bite. From that

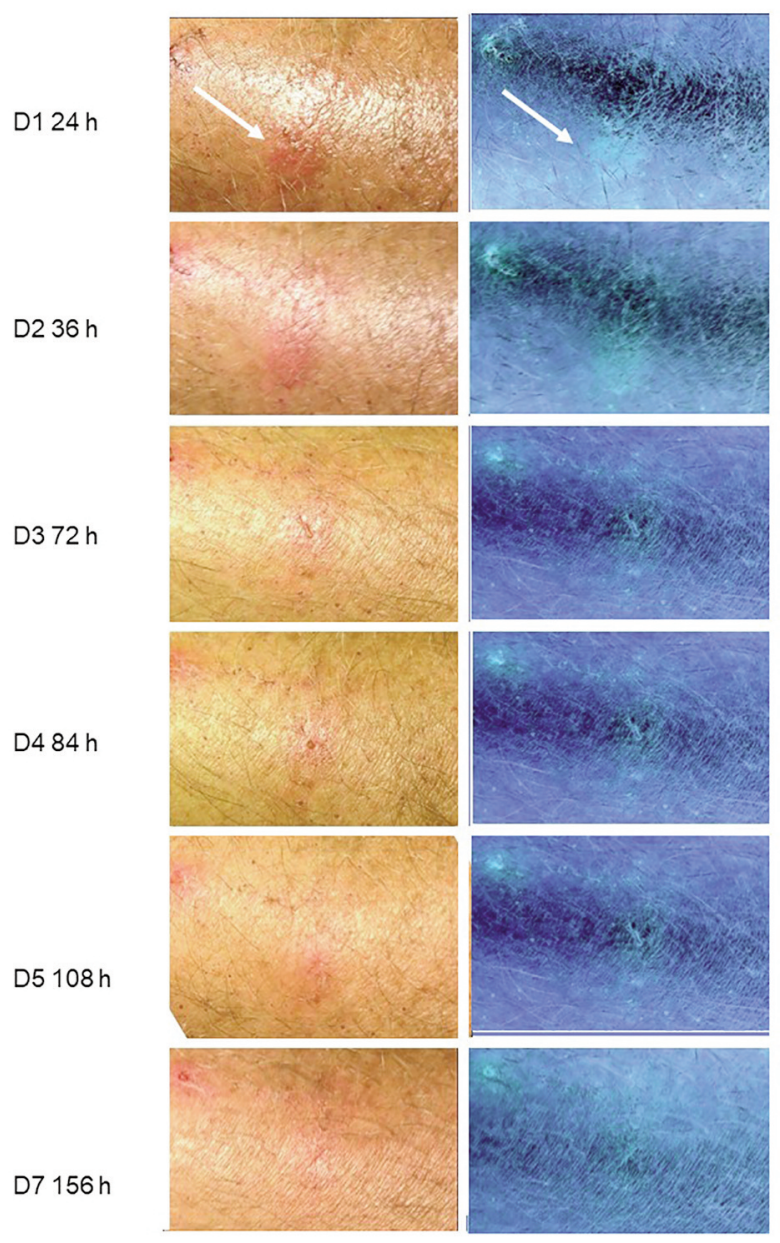

Figure 1. Time-dependent resolution of a brown recluse spider bite in the right forearm by topical DEA. The pictures obtained between $24 \mathrm{~h}$ (Day1) and 156 h (Day 7) are shown as color images (left column) and inverted color images (right column). The site of the spider bite is marked with an arrow.

time point there was a gradual decrease of the redness, pain and swelling that was entirely resolved by Day 14. A bar chart (Figure 2C) illustrates relative sizes of the red areas of the neck around the bite site over the treatment course with topical DEA for 14 days. The $\mathrm{x}$-axis shows the elapsed time since the envenomation event in hours and days.

DEA inhibited hemolysis induced by L. reclusa venom or recombinant PLD from $L$. intermedia. Since PLD in the recluse venom is the key player responsible for hemolysis, we examined whether DEA could affect hemolysis induced by the L. reclusa venom (LOX) or the recombinant PLD (rPLD) from the closely related $L$. intermedia. In the latter case, red blood cells hemolysis was used as a surrogate of PLD enzymatic activity. 

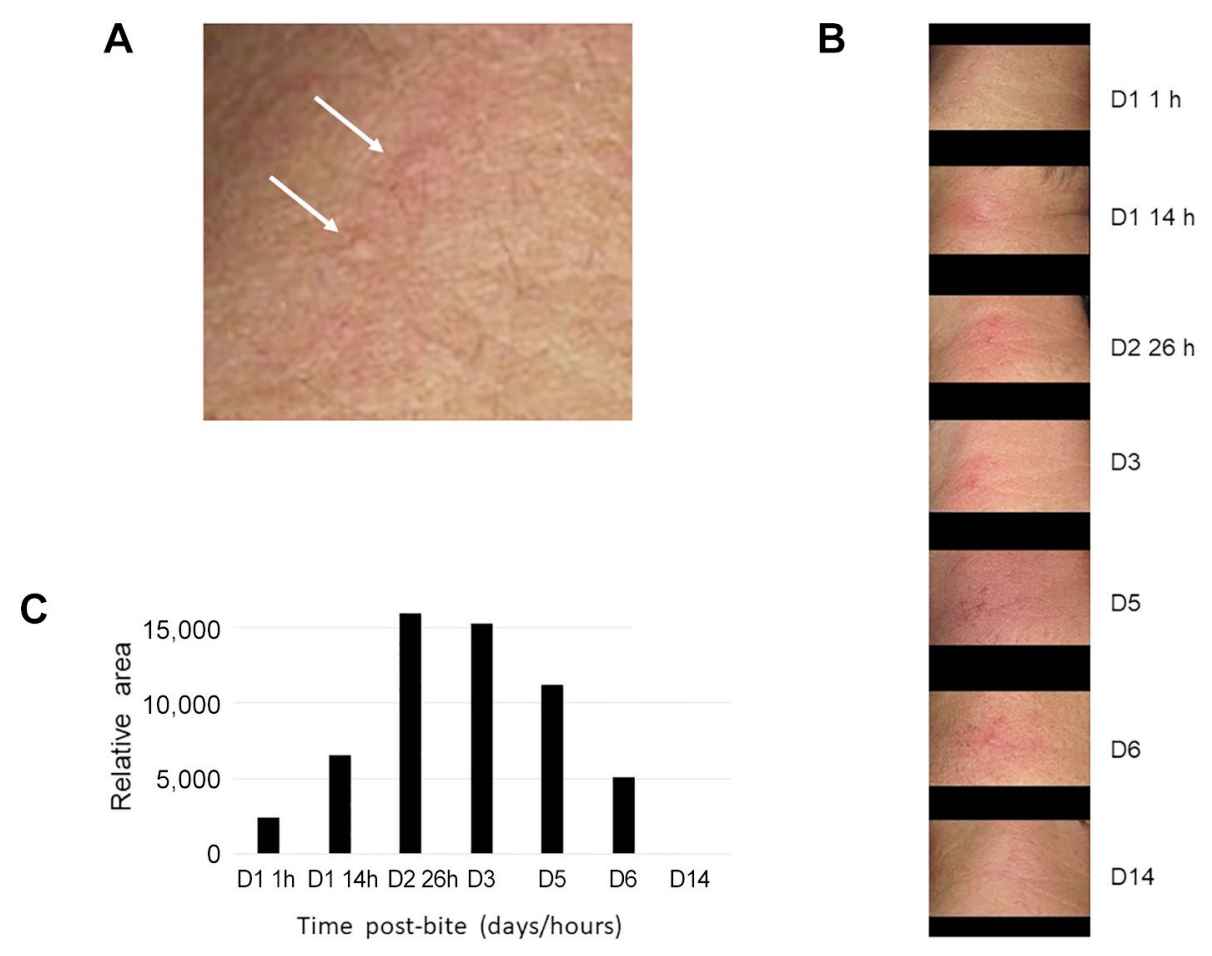

Figure 2. Time-dependent resolution of a brown recluse spider bite in the neck by topical DEA. A) Magnified image of the neck area with two bites on Day 1 (D1) hour 1 (H1). Arrows point out to the bite sites. B) Time course images of the bite area and time-dependent resolution of a brown recluse spider bite over a treatment course with topical DEA.C) Quantitative analysis of the affected bite area. A bar chart shows relative size of the arm area at the bite site over the treatment with topical DEA for 14 days. The $x$-axis shows the elapsed time since the envenomation event in days and hours as applicable.

As shown in Figure 3A, in a 60 min assay both Triton X and DEA demonstrated a dose-dependent effect on hemolysis induced by LOX or rPLD normalized to the same protein concentration. DEA alone even at the highest examined concentration of $5 \%$ corresponding to $122 \mathrm{mM}$ was not hemolytic as demonstrated by the optical density at $400 \mathrm{~nm}$ of the erythrocyte suspension (see dotted line in Figure 3A). The dose response of hemolysis due to Triton $\mathrm{X}$ was apparently linear (Figure 3A). In contrast, DEA exhibited a non-linear dose response against rPLD and LOX with complete inhibition of the hemolytic activity at $122 \mathrm{mM}$ and $5 \mathrm{mM}$ DEA, respectively. These findings are not surprising as LOX was less hemolytic than rPLD.

Next, we examined if the order of adding test samples to the assay mixture could affect the extent of hemolysis (Figure 3B). As before, Triton X100 was used as a positive control and the total incubation time was $60 \mathrm{~min}$. When LOX $(75 \mathrm{mg} / \mathrm{ml})$ was added to the erythrocyte suspension, hemolysis was moderately decreased by $22 \%$. On the other hand, when erythrocytes were first preincubated with DEA and subsequently exposed to LOX, or when DEA was first preincubated with LOX and then the mixture was added to the erythrocyte suspension, hemolysis was completely inhibited.
The results gave us an early insight into the possible mechanism of action of DEA. Preincubation of DEA with erythrocytes could deplete some DEA due to its binding to plasma membrane of erythrocytes. In consequence, less DEA was available for the inhibition of hemolysis induced by either rPLD or LOX. Preincubation of DEA with either rPLD or LOX apparently affected PLD structure in a way that resulted in the inhibition of enzymatic activity. We hypothesize that DEA might interact with lipophilic regions of PLD.

Given that the hemolytic activity of LOX is lower than that of rPLD, it is not surprising that the weaker PLD enzymatic activity in the LOX preparation was inhibited by DEA still remaining in the solution. This was not the case with rPLD. The differences in hemolytic activities of the neat venom and rPLD may be ascribable to species differences. L. reclusa shares $85 \%$ amino acid sequence homology with $L$. intermedia but neither preparation was normalized to PLD activity.

DEA and related azelates inhibited hemolysis induced by honey bee venom. We investigated if DEA could also inhibit hemolysis induced by venoms of organisms other than brown 


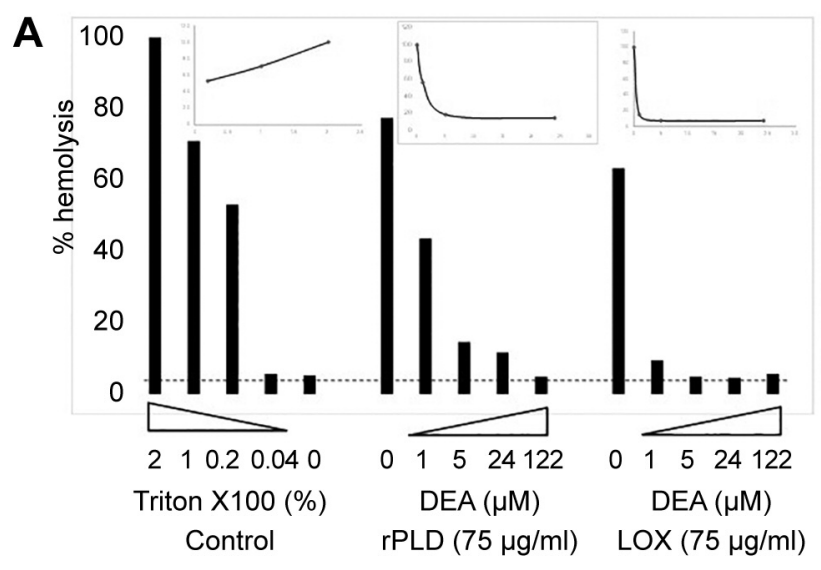

B

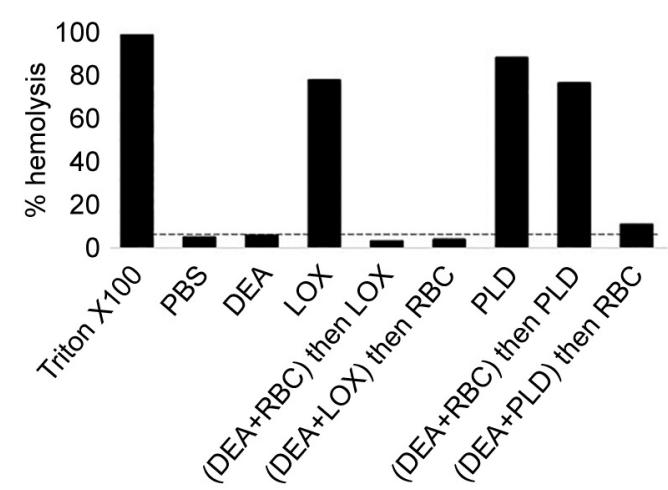

Figure 3. DEA inhibits hemolysis induced by brown recluse spider venom and recombinant phospholipase D. A) DEA dose effect on hemolysis induced by rPLD or brown recluse spider venom (LOX) compared to Triton X-100 control. Insets show the same data plotted in a linear scale of concentrations of DEA or Triton to underscore differences in the dose responses. Dotted line: hemolysis in negative control (PBS). B) The order of reagents added to the hemolysis assay reaction mixture affects the extent of hemolysis. Dotted line: hemolysis in negative control (PBS).

spiders and used water moccasin snake and honey bee as representative species. Preliminary tests showed that the snake venom had no measurable hemolytic activity. Since bee venom was highly hemolytic, we used it to evaluate the effects of DEA and six related azelates (all at 5\%) in the hemolysis assay. As shown in Figure 4, with the data corrected for background hemolysis caused by the esters alone, all azelates inhibited bee venom-induced hemolysis to a different degree. DEA, DiPA, DiBU and D1PA suppressed hemolysis by approximately 20\%, DMA was ineffective, whereas DCHA increased hemolysis by $20 \%$. The differences between DEA and DMA, D3PA or DCHA were statistically significant. The results are reminiscent of distinct effects of the azelates on plasma membrane fluidity and secreted cytokine patterns (15).

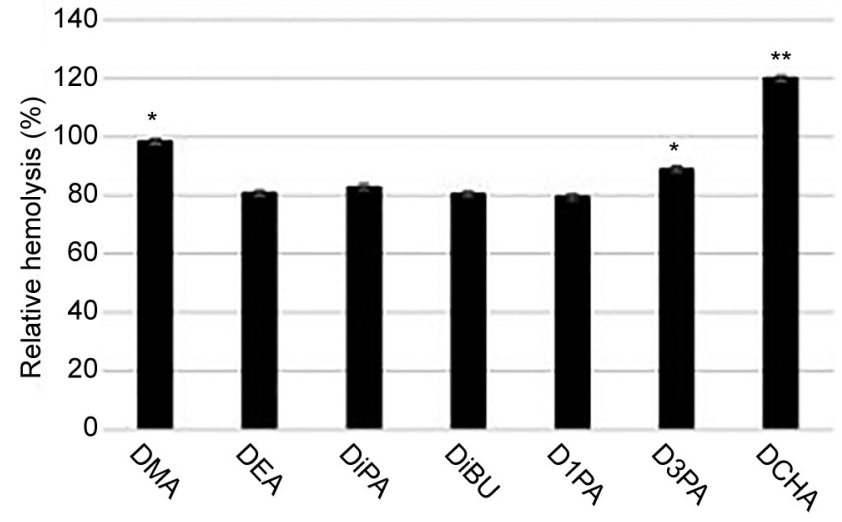

Figure 4. Effects of DEA and related azelates on hemolysis and enzymatic activity of secretory phospholipase A2 activity in bee venom. Hemolysis induced by bee venom in human erythrocytes was measured in the presence DEA and related azelates (all at 5\%). DMA: Dimethyl azelate; DiPA: di-isopropyl azelate; DiBU: di-isobutyl azelate; D1PA: di-(1-pentyl) azelate; D3PA: di-(3-pentyl) azelate; DCHA: dicyclohexyl azelate. The extent of hemolysis measured in the presence of DEA was defined as $100 \%$.

DEA inhibited phospholipase A2 activity in honey bee and moccasin snake venoms and in human urine. Since DEA inhibited rPLD, we examined if the ester could affect enzymatic activities of other members of phospholipase family. We tested honey bee and water moccasin snake venoms containing high levels of secretory phospholipase A2 (sPLA2), which promotes pain and inflammation in mammals (20). Normal human urine with intrinsic low levels of sPLA2 was also included in the study.

Figure 5 shows the effect of DEA on sPLA2 activities in the bee and snake venoms. First, we examined the dose effect of DEA on sPLA2 activities following a $15 \mathrm{~min}$ preincubation of the venoms with DEA. Initial velocities of sPLA2 activity in A. piscivoris venom were linear over the first $5 \mathrm{~min}$ of the assay at the examined concentrations of DEA up to $50 \%$ (Figure $5 \mathrm{~A}$ ) and the dose response was biphasic (Figure 5B). Qualitatively similar results were obtained using bee venom (Figure 5C and D) but the extent of sPLA2 inhibition was lower than that of the snake venom in the 15-min incubation regimen. When the incubation time was extended to $60 \mathrm{~min}, 50 \%$ DEA inhibited $>90 \%$ sPLA2 in either venom.

In human urine, preincubation with $20 \%$ DEA for 15 min inhibited sPLA2 by $53 \%$. Due to low enzymatic activity of urine aPLA2 the dose response of DEA was not measured.

Overall, DEA inhibited sPLA2 activity in the examined specimens with a non-linear dose response. The extent of inhibition was time-dependent and increased over time. 

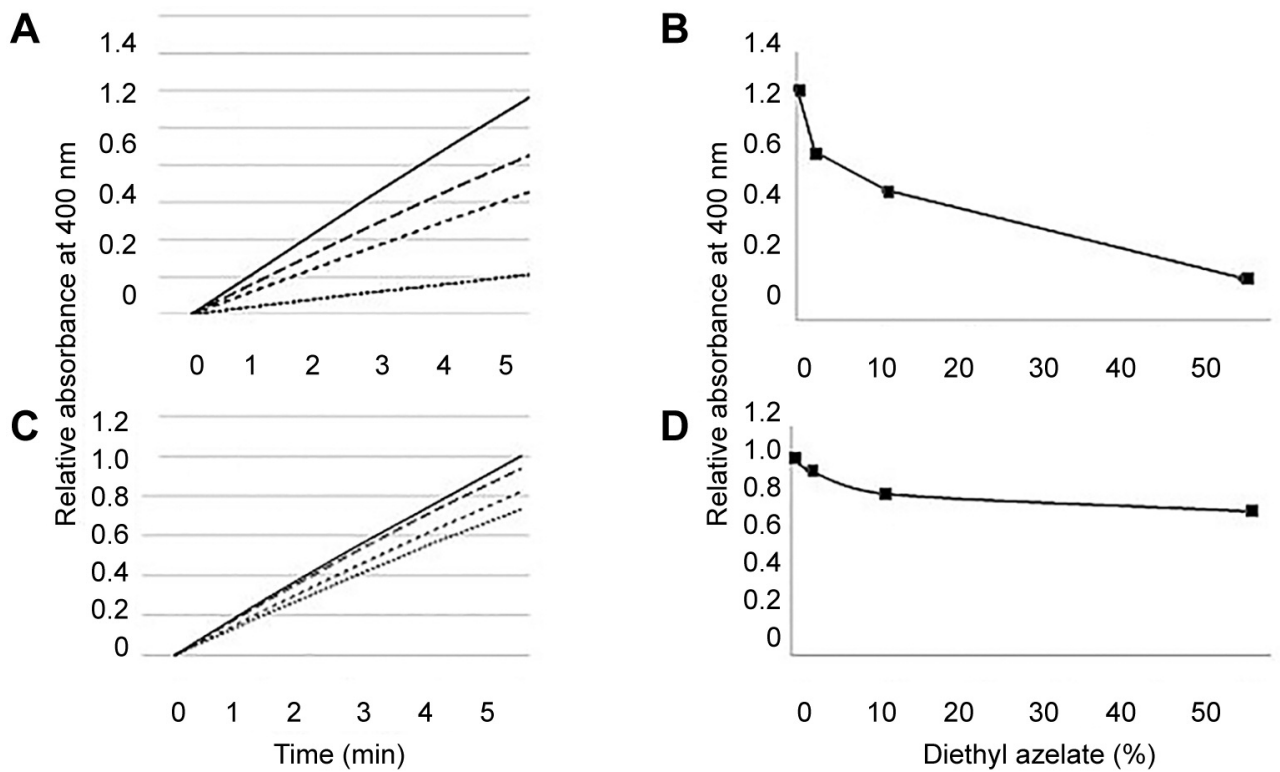

Figure 5. Effects of DEA on enzymatic activity of secreted phospholipase A2 activity in bee and snake venoms. A): Initial velocities of sPLA2 in snake venom in the presence of DEA measured as relative absorbance at $400 \mathrm{~nm}$. Solid line; untreated control. Dashed lines of increasing density correspond to 2,10 , and $50 \%$ DEA. B) initial velocities of SPLA2 activity in snake venom are plotted against DEA concentration. C) Initial velocities of sPLA2 in bee venom in the presence of DEA measured as relative absorbance at $400 \mathrm{~nm}$. Solid line; untreated control. Dashed lines of increasing density starting from the top correspond to 2, 10, and 50\% DEA. D) initial velocities of sPLA2 activity in bee venom are plotted against DEA concentration.

\section{Discussion}

This manuscript describes potential application of DEA as a novel drug for brown recluse spider bite. The project was enabled by an accidental recluse spider bite that necessitated prompt self-treatment with topical DEA. Based on the published data on favorable safety profiles of azelates including our clinical study whereby DEA was orally administered daily for 3 weeks $(15,17)$ we had surmised that the risk of using topical DEA was low.

Interestingly, a report on self-treatment of multiple brown recluse spider bites with topical 50\% trichloroacetic acid was presented as a new therapy (21). This report along with our work adds to a long history of self-experimentation (22) including John Scott Haldane's work on respiration, Joseph Goldberger's studies on dietary deprivations and Werner Forssmann's heart cathetherization that brought the investigator a Nobel Prize in medicine.

There are no specific drugs for brown recluse envenomation. An approved treatment using polyvalent horse antiserum is limited to a few Latin America countries and carries a risk of known side effects of antivenom serum therapies. To the best of our knowledge, small molecule treatments beyond tetracycline which is not a specific or curative treatment for the bite, are at the experimental stage. The current status of trichloroacetic acid for treatment of brown recluse bite is uncertain.
There is an urgent need for a safe and effective therapy of the brown recluse envenomation, especially its systemic effects. DEA is a viable novel treatment for the brown recluse spider bite because of an early indication of its efficacy in this study and an excellent safety profile of the ester. DEA and other azelates are natural metabolites in humans and other mammals. Azelates are also present in grains and grain-derived products including liquors, and in fermented foods due to bacterial degradation of acyl glycerol fatty acids and esterification of the medium chain fatty acids (15). Although the topical safety of DEA has not yet been evaluated, it is known that the azelates are rapidly hydrolyzed to azelaic acid (23) and the latter that has been used in topical applications for half a century (24).

What are the advantages of DEA as a drug for the brown recluse spider bite? Unlike current efforts in loxoscelism drug development including repurposing of an antibiotic and targeted approaches involving inhibition of PLD or complement (25), DEA is expected to strike loxoscelism at multiple levels due to its broad spectrum of activities (15, 17). DEA apparently affects not only the enzyme (PLD) itself but also its substrates (membrane phospholipids) and the host immune response to the venom.

More specifically, DEA directly affects PLD enzymatic activity in the brown recluse venom although the mechanism of the inhibition remains to be elucidated. The time- and dosedependent effects of DEA on hemolysis (as well as sPLA2 
activity) are both non-linear. This suggests a physicochemical effect rather than simple inhibition of an enzyme by a parent compound and its metabolite (26). We have also noted nonlinear dose effects of DEA in other studies (15).

DEA should not be viewed as a mere inhibitor of PLD because the ester could affect interactions between PLD and its substrates, membrane phospholipids, by simple changes in the plasma membrane fluidity. Certain products of PLD activity, such as cyclic phosphates exemplified by cyclic phosphatidic acid are of particular interest as the initiators of pathological events in loxoscelism (7). Cyclic phosphatidic acid acts act as a second messenger and inhibits peroxisome proliferatoractivated receptor (PPAR) gamma, a nuclear receptor that plays role in cell proliferation, apoptosis, inflammation, energy homeostasis and metabolic functions. Activation of PPAR gamma causes insulin sensitization and enhances glucose metabolism (27). Since DEA mitigates insulin resistance in humans (17) it may also reverse pathological effects of cyclic phosphatidic acid signaling. Interestingly, azelaic acid induces PPAR gamma mRNA and its transcriptional activity and modulates inflammatory response in human keratinocytes (28). DEA hydrolysis to azelaic acid may be therefore a desirable feature of DEA metabolism in the treatment of loxoscelism.

DEA can be viewed as a systemic modulator of the host response to the venom with a range of anti-inflammatory and immunomodulatory properties. We have demonstrated in vitro (15) that DEA downregulated matrix metalloproteinases which are also abundant in the recluse spider venom. In these models DEA also suppressed pro-inflammatory interleukins IL-6 and IL-8, CXCL1/GRO-alpha and CCL2/MCP-1, all of which are upregulated in loxoscelism (9).

Our data suggest that DEA might control pain associated with the recluse bite (29). The pain reaction to the bite results from an inflammatory response mediated by arachidonic acid, which is freed from plasma membrane phospholipids by PLA2. Secretory PLA2 is ubiquitous in venoms of arachnids $(14,30)$. Although not yet described in brown recluse venom, PLA2 might be present in this venom based on a biochemical clue, arachidonic acid. Given inhibition of sPLA2 in our in vitro work, we suggest that DEA may also inhibit unspecified PLA2(s) in the venom resulting in control of pain.

Modulation of pain due to recluse spider bite may also result from membrane fluidizing properties of DEA. In the past we have shown that DEA disrupted cholera toxin complex formation and/or its binding to monosialotetrahexosylganglioside 1 (GM1)enriched lipid rafts in human blood cells (15). Surprisingly, inhalational anesthetics also disrupt GM1 rafts by increasing plasma membrane fluidity and initiate a cascade of events leading to pain mitigation and unconsciousness (31). Since even sub-anesthetic concentrations of inhalational anesthetics can reduce pain (32) topical DEA may also bring pain relief in loxoscelism.
In conclusion, DEA appears to be a promising new therapy for the brown recluse spider bite. Our findings warrant further development of the molecule as an orphan drug for loxoscelism. In addition, a broad spectrum of phospholipase inhibitory activity by DEA suggests that the compound may find application in the treatment for select envenomations and human diseases that elaborate PLDs and PLA2s.

\section{Conflicts of Interest}

EI and RTS are the owners and officers of New Frontier Labs, LLC, the sponsor of all studies described in the manuscript.

\section{Authors' Contributions}

Both Authors contributed equally to the study design, experimental work, data analysis, and writing of the manuscript.

\section{References}

1 Vetter RS: The distribution of brown recluse spiders in the southeastern quadrant of the United States in relation to loxoscelism diagnoses. South Med J 102(5): 518-522, 2009. PMID: 19373143. DOI: 10.1097/SMJ.0b013e3181a0abb3

2 Swanson DL and Vetter RS: Bites of brown recluse spiders and suspected necrotic arachnidism. N Engl J Med 352(7): 700-707, 2005. PMID: 15716564. DOI: 10.1056/NEJMra041184

3 Stoecker WV, Vetter RS and Dyer JA: NOT RECLUSE-A mnemonic device to avoid false diagnoses of brown recluse spider bites. JAMA Dermatol 153(5): 377-378, 2017. PMID: 28199453. DOI: 10.1001/jamadermatol.2016.5665

4 Mowry JB, Spyker DA, Brooks DE, McMillan N and Schauben JL: 2014 annual report of the American Association of Poison Control Centers' National Poison Data System (NPDS): 32nd annual report. Clin Toxicol (Phila) 53(10): 962-1147, 2015. PMID: 26624241. DOI: 10.3109/15563650.2015.1102927

5 Forrester JD, Forrester JA, Tennakoon L and Staudenmayer K: Mortality, hospital admission, and healthcare cost due to injury from venomous and non-venomous animal encounters in the USA: 5-year analysis of the National Emergency Department Sample. Trauma Surg Acute Care Open 3(1): e000250, 2018. PMID: 30623028. DOI: 10.1136/tsaco-2018-000250

6 Robinson JR, Kennedy VE, Doss Y, Bastarache L, Denny J and Warner JL: Defining the complex phenotype of severe systemic loxoscelism using a large electronic health record cohort. PLoS One 12(4): e0174941, 2017. PMID: 28422977. DOI: 10.1371/ journal.pone. 0174941

7 Lajoie DM, Zobel-Thropp PA, Kumirov VK, Bandarian V, Binford GJ and Cordes MH: Phospholipase D toxins of brown spider venom convert lysophosphatidylcholine and sphingomyelin to cyclic phosphates. PLoS One 8(8): e72372, 2013. PMID: 24009677. DOI: 10.1371/journal.pone.0072372

8 Gremski LH, da Justa HC, da Silva TP, Polli NLC, Antunes BC, Minozzo JC, Wille ACM, Senff-Ribeiro A, Arni RK and Veiga SS: Forty years of the description of brown spider venom Phospholipases-D. Toxins (Basel) 12(3): 164, 2020. PMID: 32155765. DOI: $10.3390 /$ toxins 12030164

9 Rojas JM, Arán-Sekul T, Cortés E, Jaldín R, Ordenes K, Orrego PR, González J, Araya JE and Catalán A: Phospholipase D from 
Loxosceles laeta spider venom induces IL-6, IL-8, CXCL1/GRO- $\alpha$, and CCL2/MCP-1 production in human skin fibroblasts and stimulates monocytes migration. Toxins (Basel) 9(4): 125, 2017. PMID: 28379166. DOI: 10.3390/toxins9040125

10 Cordes MHJ and Binford GJ: Evolutionary dynamics of origin and loss in the deep history of phospholipase D toxin genes. BMC Evol Biol 18(1): 194, 2018. PMID: 30563447. DOI: 10.1186/s12862-018-1302-2

11 Selvy PE, Lavieri RR, Lindsley $\mathrm{CW}$ and Brown HA: Phospholipase D: enzymology, functionality, and chemical modulation. Chem Rev 111(10): 6064-6119, 2011. PMID: 21936578. DOI: $10.1021 / \mathrm{cr} 200296 \mathrm{t}$

12 Lajoie DM and Cordes MH: Spider, bacterial and fungal phospholipase D toxins make cyclic phosphate products. Toxicon 108: 176-180, 2015. PMID: 26482933. DOI: 10.1016/ j.toxicon.2015.10.008

13 Tambourgi DV, Gonçalves-de-Andrade RM and van den Berg CW: Loxoscelism: From basic research to the proposal of new therapies. Toxicon 56(7): 1113-1119, 2010. PMID: 20144641. DOI: $10.1016 /$ j.toxicon.2010.01.021

14 Dennis EA: Introduction to thematic review series: Phospholipases: Central role in lipid signaling and disease. J Lipid Res 56(7): 1245-1247, 2015. PMID: 26031662. DOI: 10.1194/jlr.E061101

15 Izbicka E, Streeper RT and Louden C: Adaptive membrane fluidity modulation: A feedback regulated homeostatic system and target for pharmacological intervention. In Vivo 35(6): 3073-3095, 2021. PMID: 34697139. DOI: 10.21873/invivo. 12603

16 Izbicka E and Streeper RT: Adaptive membrane fluidity modulation: A feedback regulated homeostatic system hiding in plain sight. In Vivo 35(6): 2991-3000, 2021. PMID: 34697130. DOI: 10.21873 /invivo.12594

17 Streeper RT, Louden C and Izbicka E: Oral azelaic acid ester decreases markers of insulin resistance in overweight human male subjects. In Vivo 34(3): 1173-1186, 2020. PMID: 32354907. DOI: 10.21873 /invivo. 11890

18 Lowe NJ: New approaches in dermatology: a clinical profile of azelaic acid. Cutis 57(1 Suppl): 7, 1996. PMID: 8654130.

19 Evans BC, Nelson CE, Yu SS, Beavers KR, Kim AJ, Li H, Nelson HM, Giorgio TD and Duvall CL: Ex vivo red blood cell hemolysis assay for the evaluation of $\mathrm{pH}$-responsive endosomolytic agents for cytosolic delivery of biomacromolecular drugs. J Vis Exp (73): e50166, 2013. PMID: 23524982. DOI: $10.3791 / 50166$

20 Zambelli VO, Picolo G, Fernandes CAH, Fontes MRM and Cury Y: Secreted phospholipases $A_{2}$ from animal venoms in pain and analgesia. Toxins (Basel) 9(12): 406, 2017. PMID: 29311537. DOI: $10.3390 /$ toxins9120406

21 Schultze C: A new therapy for brown recluse spider bites. Emerg Med News 42(3): 28-29, 2020. DOI: 10.1097/01.EEM. 0000657636.41011.d9

22 Altman LK: Who goes first? The story of self-experimentation in medicine. $1^{\text {st }}$ ed. New York, Random House, 1987.
23 Charnock C, Brudeli B and Klaveness J: Evaluation of the antibacterial efficacy of diesters of azelaic acid. Eur J Pharm Sci 21(5): 589-596, 2004. PMID: 15066659. DOI: 10.1016/j.ejps. 2003.12.006

24 Schulte BC, Wu W and Rosen T: Azelaic acid: Evidence-based update on mechanism of action and clinical application. J Drugs Dermatol 14(9): 964-968, 2015. PMID: 26355614.

25 Gehrie EA, Nian H and Young PP: Brown Recluse spider bite mediated hemolysis: clinical features, a possible role for complement inhibitor therapy, and reduced RBC surface glycophorin A as a potential biomarker of venom exposure. PLoS One 8(9): e76558, 2013. PMID: 24086749. DOI: 10.1371/ journal.pone. 0076558

26 Rowland Yeo K, Jamei M, Yang J, Tucker GT and RostamiHodjegan A: Physiologically based mechanistic modelling to predict complex drug-drug interactions involving simultaneous competitive and time-dependent enzyme inhibition by parent compound and its metabolite in both liver and gut - the effect of diltiazem on the time-course of exposure to triazolam. Eur $\mathbf{J}$ Pharm Sci 39(5): 298-309, 2010. PMID: 20025966. DOI: 10.1016/j.ejps.2009.12.002

27 Ferré P: The biology of peroxisome proliferator-activated receptors: relationship with lipid metabolism and insulin sensitivity. Diabetes 53 Suppl 1: S43-S50, 2004. PMID: 14749265. DOI: $10.2337 /$ diabetes.53.2007.s43

28 Mastrofrancesco A, Ottaviani M, Aspite N, Cardinali G, Izzo E, Graupe K, Zouboulis CC, Camera E and Picardo M: Azelaic acid modulates the inflammatory response in normal human keratinocytes through PPARgamma activation. Exp Dermatol 19(9): 813-820, 2010. PMID: 20545756. DOI: 10.1111/j.16000625.2010.01107.x

29 Streeper RT, Izbicka E, New Frontier Labs, LLC, assignee. US Provisional Patent Application 2020. Dicarboxylic acid esters for inducing an analgesic effect. Serial No. 63/126,387.

30 Estrada-Gomez S, Muñoz LJ, Lanchero P and Latorre CS: Partial characterization of venom from the Colombian spider Phoneutria Boliviensis (Aranae:Ctenidae). Toxins (Basel) 7(8): 2872-2887, 2015. PMID: 26264023. DOI: 10.3390/toxins7082872

31 Pavel MA, Petersen EN, Wang H, Lerner RA and Hansen SB: Studies on the mechanism of general anesthesia. Proc Natl Acad Sci U.S.A. 117(24): 13757-13766, 2020. PMID: 32467161. DOI: 10.1073/pnas.2004259117

32 Klomp T, van Poppel M, Jones L, Lazet J, Di Nisio M and Lagro-Janssen AL: Inhaled analgesia for pain management in labour. Cochrane Database Syst Rev (9): CD009351, 2012. PMID: 22972140. DOI: 10.1002/14651858.CD009351.pub2

Received October 28, 2021

Revised November 14, 2021

Accepted November 15, 2021 